\title{
Tinea Incognita following the Use of an Antipsoriatic Gel
}

\author{
Michela Starace Aurora Alessandrini Bianca Maria Piraccini \\ Division of Dermatology, Department of Experimental, Diagnostic and Specialty Medicine, University of Bologna, \\ Bologna, Italy
}

\section{Established Facts}

- Tinea incognita is a variant of skin fungal infection.

- Topical application of steroids reduces inflammatory signs in tinea incognita.

\section{Novel Insights}

- Tinea pedis should be ruled out in patients treated with topical steroids in order to prevent spreading of a nondiagnosed pre-existing skin infection.

\section{Key Words}

Tinea incognita - Topical steroid · Calcipotriol .

Self-administered therapy

\section{Abstract}

Tinea incognita is a dermatophyte infection of the skin whose clinical presentation has been modified by the misuse of steroids or, as has been described recently, calcineurin inhibitors. We report a case of pustular psoriasis treated with an antipsoriatic cream, composed of topical steroids and a vitamin D3 derivative, which gave rise to a tinea incognita. Our case underlines that clinical features of fungal infection can be modified by the increasing use of self-prescribed topical therapies, usually applied to treat incorrectly 'self-diag- nosed' diseases. Moreover, we suggest that a mycological examination should be performed in every atypical presentation of skin lesion, and to rule out tinea pedis, in any disease of the feet that requires topical application of steroids.

(c) 2015 S. Karger AG, Basel

\section{Introduction}

Tinea incognita is a dermatophyte infection of the skin whose clinical presentation has been modified by the misuse of steroids or, as has been reported recently, calcineurin inhibitors [1, 2]. It was first described in 1968 by Ive and Marks [3]. For dermatologists, the diagnosis is often difficult to establish.

\section{KARGER 125}

(c) 2015 S. Karger AG, Basel

2296-9195/15/0013-0123\$39.50/0

E-Mail karger@karger.com

www.karger.com/sad
Bianca Maria Piraccini, Division of Dermatology

Department of Experimental, Diagnostic and Specialty Medicine

University of Bologna, Via Massarenti 1

IT-40138 Bologna (Italy)

E-Mail biancamaria.piraccini@unibo.it 


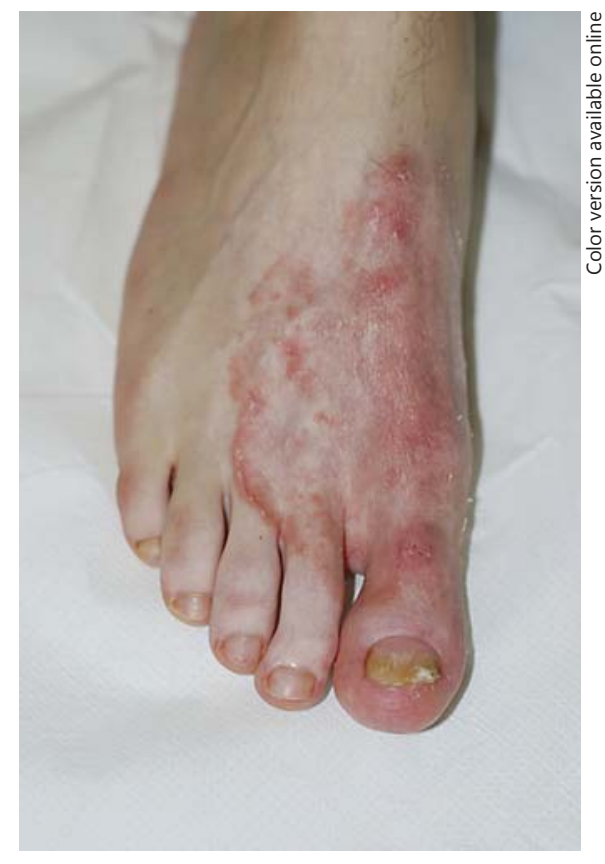

Fig. 1. Tinea incognita due to topical application of a combination of calcipotriol monohydrate and betamethasone dipropionate. An erythematous area with peripheral scaling involving a large part of the dorsum of the foot is seen.

We report a case of pustular psoriasis treated with an antipsoriatic cream, composed of topical steroids and a vitamin D3 derivative, which gave rise to a tinea incognita.

\section{Case Report}

A 17-year-old male was referred to our Department of Dermatology for a 2-month history of a nail disorder of the first left toenail, presenting as painful onycholysis with pustules and crusts of the nail bed and hyponychium. Mycology was negative and a nail bed biopsy confirmed our suspicion of pustular psoriasis. A combination of topical corticosteroid and calcipotriol therapy in gel formulation to be applied once daily was prescribed. At the follow-up visit, after 2 months, the nail plate was growing well and the periungual tissues did not show inflammatory changes. Therapy was continued with a twice weekly application. After 2 weeks, the patient noticed a pruritic erythematous area on the first interdigital space and started to apply the combination of topical calcipotriol monohydrate and betamethasone dipropionate twice daily on a wider skin area. Itching disappeared, but the skin lesion progressively enlarged. At the time of examination, 2 months after the first visit, the dorsum of the foot presented a wide erythematous area, with well demarcated scaling margins and some follicular papules (fig. 1). No lesions were identified elsewhere on the skin and toe webs. Direct examination of skin scrapings with $20 \%$ potassium hydroxide preparation $(\mathrm{KOH})$ showed fungal hyphae, and Trichophyton rubrum was isolated in Sabouraud dextrose agar culture. From these findings, we made the diagnosis of tinea incognita. Systemic treatment with terbinafine $(250 \mathrm{mg}$ /daily) for 2 weeks and topical therapy with $1 \%$ ciclopiroxolamine cream applied twice daily on the affected area of the foot was prescribed, while the calcipotriol monohydrate and betamethasone dipropionate gel was discontinued. A complete resolution of itching and erythema occurred after 4 weeks of treatment, with negative mycology.

\section{Discussion}

Fungal skin infections affect $10-20 \%$ of the world population [4] and traditionally appear as oval eruptions with fine scales and erythematous border. Tinea incognita accounts for approximately $40 \%$ of tinea infections [5] and usually develops for the erroneous administration of corticosteroids, prescribed on the basis of preexisting pathologies or wrong diagnoses [6]. In particular, topical corticosteroids suppress the fungus-induced local immunity, both innate, i.e. beta-defensins, and acquired, including $\mathrm{T}$ cell-mediated immune mechanisms [7].

The immunosuppressive activity of the topical immunomodulators pimecrolimus and tacrolimus can also be responsible for tinea incognita, as has been described by several recent reports $[2,6,8,9]$. The clinical diagnosis of tinea incognita is often missed or delayed, even by dermatologists, and the infection slowly spreads with scarce subjective and objective symptoms.

Our patient was affected by pustular psoriasis of the left great toenail involving the nail and distal pulp, and a topical combination of calcipotriol monohydrate and betamethasone dipropionate gel was applied on the nail bed and periungual skin. We speculate that the patient was also affected by tinea pedis interdigitalis, which appeared in the first interdigital space and was considered as an extension of psoriasis and treated accordingly. This led to spreading of the infection with development of tinea incognita.

The present case underlines that physicians should keep in mind that clinical features of fungal infection can be modified by the increasing use of self-prescribed topical therapies, usually applied to treat incorrectly 'selfdiagnosed' diseases [10]. In a large Italian study, Romano et al. [11] stated that the most frequent skin pathologies mimicking tinea incognita are eczema, impetigo, lupus erythematosus, rosacea and psoriasis. They sug- 
gest that a mycological examination should be performed in every atypical presentation of the above diseases. A further suggestion, which comes from our case, is to perform mycology to rule out tinea pedis in any disease of the feet that requires topical application of steroids.

\section{Statement of Ethics}

This study has been approved by the institute's committee on human research. Patient consent was obtained.

\section{Disclosure Statement}

The authors have no conflicts of interest to disclose.

\section{References}

1 Wacker J, Durani BK, Hartschuh W: Bizarre annular lesion emerging as tinea incognito. Mycoses 2004;47:447-449.

2 Crawford KM, Bostrom P, Russ B, Boyd J: Pimecrolimus-induced tinea incognito. Skinmed 2004;3:352-353.

3 Ive FA, Marks R: Tinea incognito. Br Med J 1968;3:149-152.

4 El-Gohary M, van Zuuren EJ, Fedorowicz Z, Burgess H, Doney L, Stuart B, Moore M, Little $\mathrm{P}$ : Topical antifungal treatments for tinea cruris and tinea corporis. Cochrane Database Syst Rev 2014;8:CD009992.
5 Segal D, Wells MM, Rahalkar A, Joseph M, Mrkobrada M: A case of tinea incognito. Dermatol Online J 2013;19:18175.

6 Rallis E, Koumantaki-Mathioudaki E: Pimecrolimus induced tinea incognito masquerading as intertriginous psoriasis. Mycoses 2008;51:71-73.

7 Verma S, Hay RJ: Topical steroid-induced tinea pseudoimbricata: a striking form of tinea incognito. Int J Dermatol 2015;54:e192e193.
8 Siddaiah N, Erickson Q, Miller G, Elston DM: Tacrolimus-induced tinea incognito. Cutis 2004;73:237-238.

9 Crawford KM, Bostrom P, Russ B, Boyd J: Pimecrolimus-induced tinea incognito. Skinmed 2004;3:352-353.

10 del Boz J, Crespo V, Rivas-Ruiz F, de Troya M Tinea incognito in children: 54 cases. Mycoses 2011;54:254-258.

11 Romano C, Maritati E, Gianni C: Tinea incognito in Italy: a 15-year survey. Mycoses 2006; 49:383-387. 